\title{
Analytical Solution vs. Numerical Solution of Heat Equation Flow Through Rod of Length 8 Units in One Dimension
}

\section{Geleta Kinkino Meyu, Kedir Aliyi Koriche}

Department of Mathematics, College of Natural and Computational Science, Ambo University, Ambo, Ethiopia

Email address:

gkinkino@gmail.com (G. K. Meyu), kediraliyi39@gmail.com (K. A. Koriche)

\section{To cite this article:}

Geleta Kinkino Meyu, Kedir Aliyi Koriche. Analytical Solution vs. Numerical Solution of Heat Equation Flow Through Rod of Length 8 Units in One Dimension. International Journal of Applied Mathematics and Theoretical Physics. Vol. 7, No. 2, 2021, pp. 53-61. doi: $10.11648 /$ j.ijamtp.20210702.12

Received: January 25, 2021; Accepted: June 7, 2021; Published: July 2, 2021

\begin{abstract}
The paper presented the basic treatment of the solution of heat equation in one dimension. Heat is a form of energy in transaction and it flows from one system to another if there is a temperature difference between the systems. Heat flow is the main concern of sciences which seeks to predict the energy transfer which may take place between material bodies as result of temperature difference. Thus, there are three modes of heat transfer, i.e., conduction, radiation and convection. Conduction can be steady state heat conduction, or unsteady state heat conduction. If the system is in steady state, temperature doesn't vary with time, but if the system in unsteady state temperature may varies with time. However, if the temperature of material is changing with time or if there are heat sources or sinks within the material the situation is more complex. So, rather than to escape all problem, we are targeted to solve one problem of heat equation in one dimension. The treatment was from both the analytical and the numerical view point, so that the reader is afforded the insight that is gained from analytical solution as well as the numerical solution that must often be used in practice. Analytical we used the techniques of separation of variables. It is worthwhile to mention here that, analytical solution is not always possible to obtain; indeed, in many instants they are very cumber some and difficult to use. In that case a numerical technique is more appropriate. Among numerical techniques finite difference schema is used. In both approach we found a solution which agrees up to one decimal place.
\end{abstract}

Keywords: Analytical Approach, Numerical Approach, Heat Equation

\section{Introduction}

The heat equation in one dimension

We begin the study of partial differential equations with the problem of heat flow in uniform bar of length 8 units situated on the $\mathrm{x}$ - axis with one end at origin and the other at $x=8$ units.

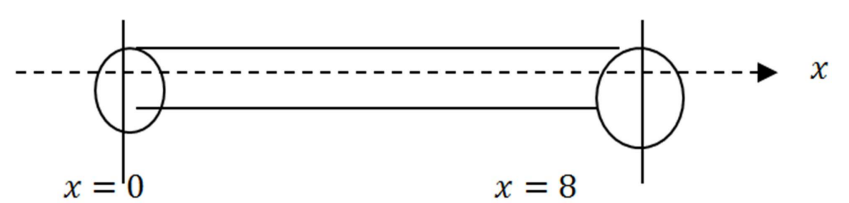

Figure 1. A uniform bar of length 8 units.

We assume that the bar is perfectly insulated except possibly at its end points and that the temperature is constant on each cross section and therefore depends only on $\mathrm{x}$ and $\mathrm{t}$.
We also assume that the thermal properties of the bar are independent of $\mathrm{x}$ and $\mathrm{t}$. The temperature $u(x, t)$ at time $\mathrm{t}$ at point $\mathrm{x}$ units from the origin satisfies the partial differential equation.

$$
u_{t}-4 u_{x x}=0 ; 0 \leq x \leq 8, t>0
$$

Subject to

$$
\begin{gathered}
u(0, t)=0=u(8, t) ; t \geq 0 \text { and } \\
u(x, 0)=4 x-\frac{x^{2}}{2} ; 0 \leq x \leq 8
\end{gathered}
$$

This is the heat equation with its boundary and initial condition which governs the heat distribution in the above uniform bar of length 8 units, see e.g. $[1,8,3]$. Having the governing equation to one dimensional heat equation, there is a need to determine its solution. 


\section{Result and Analysis}

\subsection{Analytical Approach}

Heat equation in one dimension can be solved by analytical approach. In order to solve, one dimensional heat equation with this method we will apply separation of variables which is the solutions to homogeneous PDE $[5,1,4$, 12]. So, consider the following heat conduction problem in a finite interval.

$$
\begin{gathered}
u_{t}-k u_{x x}=0 \leq x \leq L, t>0 \\
u(0, t)=u(L, t)=0 t \geq 0 \\
u(x, 0)=f(x) 0 \leq x \leq L
\end{gathered}
$$

Where, $f$ is a given initial condition, and $\mathrm{k}$ is positive constant. We assume the compatibility condition $f(0)=$ $f(L)=0$. The solution has special form:

$$
u(x, t)=\mathrm{X}(x) \mathrm{T}(t)
$$

By differentiating the separated solution (4) once with respect $\mathrm{t}$, twice with respect to $\mathrm{X}$ and $\lambda$ be a separation constant such that.

$$
\frac{\mathrm{T}_{\mathrm{x}}}{k \mathrm{~T}}=\frac{\mathrm{X}_{\mathrm{xx}}}{\mathrm{X}}=-\lambda
$$

Thus, equation (5) leads to the following system of DEs:

$$
\begin{aligned}
& \frac{d^{2} x}{d x}+\lambda x=00 \leq \mathrm{x} \leq \mathrm{L} \\
& \frac{d \mathrm{~T}}{d t}+\lambda k \mathrm{~T}=0 t>0
\end{aligned}
$$

So, the general solution for $\lambda>0$ is

$$
\mathrm{X}(x)=\alpha \cos (\sqrt{\lambda} x)+\beta \sin (\sqrt{\lambda} x)
$$

Substituting this solution in to the boundary condition $\sin (\sqrt{\lambda} L)=0$, Therefore, $\sqrt{\lambda} L=n \pi$ where $\mathrm{n}$ is positive integer. The corresponding Eigen functions are $x(x)=$ $\sin \frac{n \pi x}{L}$, see, e.g. $[5,11,14,17]$. It is convenient to use the notations.

$$
\begin{gathered}
x_{n}(x)=\sin \frac{n \pi}{L}, \lambda_{n}=\left(\frac{n \pi}{L}\right)^{2} n=1,2,3, \ldots \\
\beta_{n}=\frac{2}{L} \int_{0}^{g} f(x) \sin \frac{n \pi}{8} x d x=\frac{2}{8} \int_{0}^{g}\left(4 x-\frac{x^{2}}{2}\right) \sin \frac{n \pi x}{8} \\
=\frac{1}{4}\left[4\left(\left.\frac{-8 x}{n \pi} \cos \frac{n \pi x}{8}\right|_{0} ^{8}+\left.\frac{8^{2}}{(n \pi)^{2}} \sin \frac{n \pi x}{8}\right|_{0} ^{8}\right)-\frac{1}{2}\left(\left.\frac{-8 x^{2}}{n \pi} \cos \frac{n \pi x}{8}\right|_{0} ^{8}+\frac{16}{n \pi}\left(\frac{8 x}{n \pi} \sin \frac{n \pi x}{8}+\frac{8^{2}}{(n \pi)^{2}} \cos \frac{n \pi x}{8}\right) \mid \begin{array}{l}
8 \\
0
\end{array}\right)\right] \\
=\frac{1}{4}\left[\left.\frac{-32 x}{n \pi} \cos \frac{n \pi x}{8}\right|_{0} ^{8}-\left.\frac{1}{2}\left(\frac{-8 x^{2}}{n \pi} \cos \frac{n \pi x}{8}+\frac{1024}{n^{3} \pi^{3}} \cos \frac{n \pi x}{8}\right)\right|_{0} ^{8}\right] \\
=\frac{1}{4}\left[\frac{-256}{n \pi} \cos n \pi+\frac{-256}{n \pi} \cos n \pi \frac{-512}{(n \pi)^{3}} \cos n \pi+\frac{512}{(n \pi)^{3}}\right]
\end{gathered}
$$

Form physical point of view it is clear that the solution of (8) most decay in time.

$$
\begin{gathered}
u_{n}(x, t)=\mathrm{X}_{n}(x) \mathrm{T}_{n}(t)=\mathrm{B}_{n} \sin \frac{n \pi x}{L} e^{-\mathrm{k}\left(\frac{n \pi}{L}\right)^{2} t} \\
\text { where } n=1,2,3, \ldots
\end{gathered}
$$

The generalized super position principle implies that the formal expression

$$
u(x, t)=\sum_{n=1}^{\infty} \beta_{n} \sin \frac{n \pi x}{L} e^{-\mathrm{k}\left(\frac{n \pi}{L}\right)^{2} t} n=1,2,3, \ldots
$$

is a natural candidate of solution for generalized solution of problem (1) up to (3). Where

$f(x)=\sum_{n=1}^{\infty} \beta_{n} \sin \frac{n \pi x}{L} n=1,2,3, \ldots$ is Fourier sine series representation of $f(x)$,.

Therefore, the Fourier coefficient are given by

$$
\beta_{n}=\frac{2}{L} \int_{0}^{g} \sin \left(\frac{n \pi x}{L}\right) f(x) d x, n=1,2,3, \ldots
$$

Now, let us solve the problem

$$
\begin{gathered}
u_{t}-4 u_{x x}=0,0 \leq x \leq 8, t>0 \\
u(0, t)=0=u(8, t), t \geq 0 \\
u(x, 0)=f(x)=4 x-\frac{1}{2} x^{2}, 0 \leq x \leq 8
\end{gathered}
$$

Using analytical approach.

Solution: using equation (9) The analytical solution is

$$
\begin{aligned}
u(x, t) & =\sum_{n=1}^{\infty} \beta_{n} \sin \frac{n \pi x}{8} e^{-4\left(\frac{n \pi}{8}\right)^{2} t} n=1,2,3, \ldots \\
& =\sum_{n=1}^{\infty} \beta_{n} \sin \frac{n \pi x}{8} e^{-\left(\frac{n \pi}{16}\right)^{2} t} \text { since }
\end{aligned}
$$




$$
=\frac{-128 \cos n \pi}{(n \pi)^{3}}+\frac{128}{(n \pi)^{3}}=\frac{128}{n^{3} \pi^{3}}(1-\cos n \pi)=\left\{\begin{array}{c}
0 \text { if } n \text { is even } \\
\frac{256}{n^{3} \pi^{3}} \text { if } n \text { is odd }
\end{array}\right.
$$

Thus, the analytical solution

$$
U(x, t)=\sum_{n=1}^{\infty} u_{n}(\mathrm{x}, \mathrm{t})=\frac{256}{\pi^{3}} \sum_{\mathrm{n}=1}^{\infty} \frac{1}{(2 \mathrm{n}-1)^{3}} \sin \frac{(2 \mathrm{n}-1) \pi \mathrm{x}}{8} e^{\frac{-(2 \mathrm{n}-1)}{16} \pi^{2} \mathrm{t}}, \mathrm{n}=1,2,3, \ldots
$$

Where $f(x)=\frac{256}{\pi^{3}} \sum_{\mathrm{n}=1}^{\infty} \frac{1}{(2 \mathrm{n}-1)^{3}} \sin \frac{(2 \mathrm{n}-1) \pi \mathrm{x}}{8}, n=1,2,3, \ldots$

If we obtained the solution by adding 8 terms

i.e., for $n=1,2,3, \ldots, 8$

$$
\begin{gathered}
u(x, 0)=f(x) \approx \frac{256}{\pi^{3}} \\
\left(\sin \frac{\pi x}{8}+\frac{1}{24} \sin \frac{3 \pi x}{8}+\frac{1}{125} \sin \frac{5 \pi x}{8}+\frac{1}{343} \sin \frac{7 \pi x}{8}+\frac{1}{729} \sin \frac{9 \pi x}{8}+\frac{1}{1331} \sin \frac{11 \pi x}{8}+\frac{1}{1297} \sin \frac{13 \pi x}{8}+\frac{1}{3375} \sin \frac{15 \pi x}{8}\right)
\end{gathered}
$$

So, by computing for $x=0,1,2,3, \ldots, 8$ successively and correcting the result up to fourth decimal places, we get

$$
\begin{gathered}
u(0,0)=0, u(1,0)=3.4979, u(2,0)=5.9987, u(3,0)=7.4989, \\
u(4,0)=7.9990, u(5,0)=7.4989, u(6,0)=5.9987, u(7,0)=3.4979,
\end{gathered}
$$

and $u(8,0)=0$. Then

$$
\begin{gathered}
u(x, t) \approx \sum_{n=1}^{8} u_{n}(\mathrm{x}, \mathrm{t}) \\
=\frac{256}{\pi^{3}}\left(\sin \frac{\pi_{\mathrm{x}}}{8} \mathrm{e} \frac{-\pi^{2} \mathrm{t}}{16}+\frac{1}{27} \sin \frac{3 \pi \mathrm{x}}{8} \mathrm{e} \frac{-9 \pi^{2}}{16} \mathrm{t}+\frac{1}{125} \sin \frac{3 \pi \mathrm{x}}{8} \mathrm{e} \frac{-25 \pi^{2}}{16} \mathrm{t}+\frac{1}{343} \sin \frac{7 \pi \mathrm{x}}{8} \mathrm{e} \frac{-49 \pi^{2}}{16} \mathrm{t} \frac{1}{729} \sin \frac{9 \pi \mathrm{x}}{8} \mathrm{e} \frac{-81 \pi^{2}}{16} \mathrm{t}\right. \\
\left.+\frac{1}{1331} \sin \frac{11 \pi \mathrm{x}}{8} \mathrm{e} \frac{-125 \pi^{2}}{16} \mathrm{t}+\frac{1}{2197} \sin \frac{13 \pi \mathrm{x}}{8} \mathrm{e} \frac{-169 \pi^{2}}{16} \mathrm{t}\right) \\
=\frac{256}{\pi^{3}}
\end{gathered}
$$

$\left(\sin \frac{\pi \mathrm{x}}{8} \mathrm{e}^{-0.6568 \mathrm{t}}+\frac{1}{27} \sin \frac{3 \pi \mathrm{x}}{8} \mathrm{e}^{-5.5516 \mathrm{t}} \frac{1}{125} \sin \frac{5 \pi \mathrm{x}}{8} \mathrm{e}^{-15.4212 \mathrm{t}}\right.$

$\left.+\frac{1}{343} \sin \frac{7 \pi \mathrm{x}}{8} \mathrm{e}^{-30.2857 \mathrm{t}}+\frac{1}{729} \sin \frac{9 \pi \mathrm{x}}{8} \mathrm{e}^{-49.9649 \mathrm{t}}+\frac{1}{1331} \sin \frac{11 \pi \mathrm{x}}{8} \mathrm{e}^{-74.6389 \mathrm{t}}+\frac{1}{2197} \sin \frac{13 \pi \mathrm{x}}{8} \mathrm{e}^{-104.2477 \mathrm{t}}+\frac{1}{3375} \sin \frac{15 \pi \mathrm{x}}{8} \mathrm{e}^{-138.7913 \mathrm{t}}\right)$

If $t=\frac{1}{8}=0.125$

$$
u(x, 0.125)=\frac{256}{\pi^{3}}
$$

$$
\begin{aligned}
\left(\sin \frac{n \pi x}{8} \mathrm{e}^{-0.0771}+\frac{1}{27}\right. & \sin \frac{3 \pi \mathrm{x}}{8} \mathrm{e}^{-0.6939} \\
& +\frac{1}{125} \sin \frac{5 \pi \mathrm{x}}{8} \mathrm{e}^{-1.9276} \\
& \left.+\frac{1}{343} \sin \frac{7 \pi \mathrm{x}}{8} \mathrm{e}^{-3.7782}+\frac{1}{729} \sin \frac{9 \pi \mathrm{x}}{8} \mathrm{e}^{-6.2456}+\frac{1}{1331} \sin \frac{11 \pi \mathrm{x}}{8} \mathrm{e}^{-9.3299}+\frac{1}{2197} \sin \frac{13 \pi \mathrm{x}}{8} \mathrm{e}^{-13.031}+\frac{1}{3375} \sin \frac{15 \pi \mathrm{x}}{8} \mathrm{e}^{-17.349}\right)
\end{aligned}
$$

Thus, we get $u(0,0.125)=0, u(1,0.125)=3.0754, u(2,0.125)=5.508$,

$$
\begin{gathered}
u(3,0.125)=7.002, u(4,0.125) \\
=7.5004, u(5,0.125)=7.002, \\
u(6,0.125)=5.5058, u(7,0.125)=3.0754 \text { and } u(8,0)=0
\end{gathered}
$$

If $t=0.25$ 


$$
\begin{gathered}
u(x, 0.25)=\frac{256}{\pi^{3}} \\
\left(\sin \frac{\pi \mathrm{x}}{8} \mathrm{e}^{-0.1542}+\frac{1}{27} \sin \frac{3 \pi \mathrm{x}}{8} \mathrm{e}^{-1.3879}+\frac{1}{125} \sin \frac{5 \pi \mathrm{x}}{8} \mathrm{e}^{-3.8553}+\frac{1}{343} \sin \frac{17 \pi \mathrm{x}}{8} \mathrm{e}^{-7.5564}+\frac{1}{729} \sin \frac{9 \pi \mathrm{x}}{8} \mathrm{e}^{-12.4912}+\frac{1}{1331} \sin \frac{11 \pi \mathrm{x}}{8} \mathrm{e}^{-18.6597}+\frac{1}{2197} \sin \frac{13 \pi \mathrm{x}}{8} \mathrm{e}^{-26.0619}+\right. \\
\left.\frac{1}{3375} \sin \frac{15 \pi \mathrm{x}}{8} \mathrm{e}^{-34.6978}\right)
\end{gathered}
$$

Thus, we get $u(0,0.25)=6.5100, u(1,0.25)=2.7799, u(2,0.25)=5.0568$,

$$
\begin{gathered}
u(3,0.25)=6.5100, u(4,0.25)=7.0016, u(2,0.25)=6.5100, \\
u(6,0.25)=5.0568, u(7,0.25)=2.7799 \text { and } u(8,0.25)=0
\end{gathered}
$$

If $t=3 / 8=0.375$

$$
\begin{gathered}
u(x, 0.375)=\frac{256}{\pi^{3}} \\
\left(\sin \frac{\pi \mathrm{x}}{8} \mathrm{e}^{-0.2313}+\frac{1}{27} \sin \frac{3 \pi \mathrm{x}}{8} \mathrm{e}^{-2.0818}+\frac{1}{125} \sin \frac{5 \pi \mathrm{x}}{8} \mathrm{e}^{-5.783}+\frac{1}{343} \sin \frac{7 \pi \mathrm{x}}{8} \mathrm{e}^{-11.3346}+\frac{1}{729} \sin \frac{9 \pi \mathrm{x}}{8} \mathrm{e}^{-18.7368}+\frac{1}{1331} \sin \frac{11 \pi \mathrm{x}}{8} \mathrm{e}^{-27.9896}+\frac{1}{2197} \sin \frac{13 \pi \mathrm{x}}{8} \mathrm{e}^{-39.0929}+\right. \\
\left.\frac{1}{3375} \sin \frac{15 \pi \mathrm{x}}{8} \mathrm{e}^{-52.0467}\right)
\end{gathered}
$$

Thus, $u(0,0.375)=0 . u(1,0.375)=2.5426, u(2,0.375)=4.6594, u(3,0.375)=6.03808$

$$
\begin{gathered}
u(4,0.375)=6.5110, u(5,0.375)=6.038088, u(6,0.375)=4.6594, u(7,0.375)=2.5426 \\
\text { and } u(8,0.375)=0
\end{gathered}
$$

If $t=\frac{4}{8}=0.5$

$$
\begin{gathered}
u(x, 0.5)=\frac{256}{\pi^{3}} \\
\left(\sin \frac{\pi \mathrm{x}}{8} \mathrm{e}^{-0.3084}+\frac{1}{27} \sin \frac{3 \pi \mathrm{x}}{8} \mathrm{e}^{-2.7758}+\frac{1}{125} \sin \frac{5 \pi \mathrm{x}}{8} \mathrm{e}^{-7.7106}+\frac{1}{343} \sin \frac{7 \pi \mathrm{x}}{8} \mathrm{e}^{-15.1128}+\frac{1}{729} \sin \frac{9 \pi \mathrm{x}}{8} \mathrm{e}^{-24.9824}+\frac{1}{1331} \sin \frac{11 \pi \mathrm{x}}{8} \mathrm{e}^{-37.3194}+\frac{1}{2197} \sin \frac{13 \pi \mathrm{x}}{8} \mathrm{e}^{-52.1238}+\right. \\
\left.\frac{1}{3375} \sin \frac{15 \pi \mathrm{x}}{8} \mathrm{e}^{-69.395}\right)
\end{gathered}
$$

Thus, Thus, $u(0,0.5)=0 . u(1,0.5)=2.3387, u(2,0.5)=4.3003, u(3,0.5)=5.5963$,

$$
\begin{gathered}
u(4,0.5)=6.0788, u(5,0.5)=5.5964, u(6,0.5)=4.3023, u(7,0.5)=2.3387 \\
\text { and } u(8,0.5)=0
\end{gathered}
$$

If $t=0.625$

$$
\left.\begin{array}{c}
u(x, 0.625)=\frac{256}{\pi^{3}} \\
\sin \frac{\pi \mathrm{x}}{8} \mathrm{e}^{-0.3855}+\frac{1}{27} \sin \frac{3 \pi \mathrm{x}}{8} \mathrm{e}^{-3.4698}+\frac{1}{125} \sin \frac{5 \pi \mathrm{x}}{8} \mathrm{e}^{-9.6382}+\frac{1}{343} \sin +\frac{1}{1331} \sin \frac{11 \pi \mathrm{x}}{8} \mathrm{e}^{-46.6493}+\frac{1}{2197} \sin \frac{13 \pi \mathrm{x}}{8} \mathrm{e}^{-65.1548} \\
+\frac{1}{3375} \sin \frac{15 \pi \mathrm{x}}{8} \mathrm{e}^{-86.7446}
\end{array}\right)
$$

Thus, $u(0,0.625)=0, u(1,0.625)=2.1577, u(2,0.625)=3.9773$,

$$
\begin{gathered}
u(3,0.625)=5.1842, u(4,0.625)=5.6057, u(5,0.625)=5.1842 \\
u(6,0.625)=3.9773, u(7,0.625)=2.1577 \text { and } u(8,0.625)=0
\end{gathered}
$$

The above solution indicates how fast heat flows in a given material. Though the solution has been obtained, it involves such an infinite series solution that analytical evaluation becomes exceedingly difficult, see [2, 7]. For such situations the most powerful approach is one based on forward time central space schema, see e.g., $[10,9,13]$.

\subsection{Numerical Approach}

The solution to one dimensional heat equation can also be solved by numerical methods which is an approximate solution. Of all the numerical methods available for the solution of the partial differential equation the method of 
finite difference is significantly used to solve heat equation $[6$, $14,16]$. In order to solve heat equation by finite difference schema let us employ the Schmidt method, see e.g $[7,15]$.

Schmidt method: Consider figure 2. i.e., a rectangular mesh in the $x-t$ plane with spacing h a long $x$ direction and $\mathrm{k}$ along time direction.

Donating a mesh point $(x, t)=(i h, j k)$ as simply $i, j$.

Note: the points of intersection of dividing line are called mesh points, nodal points or grid points.

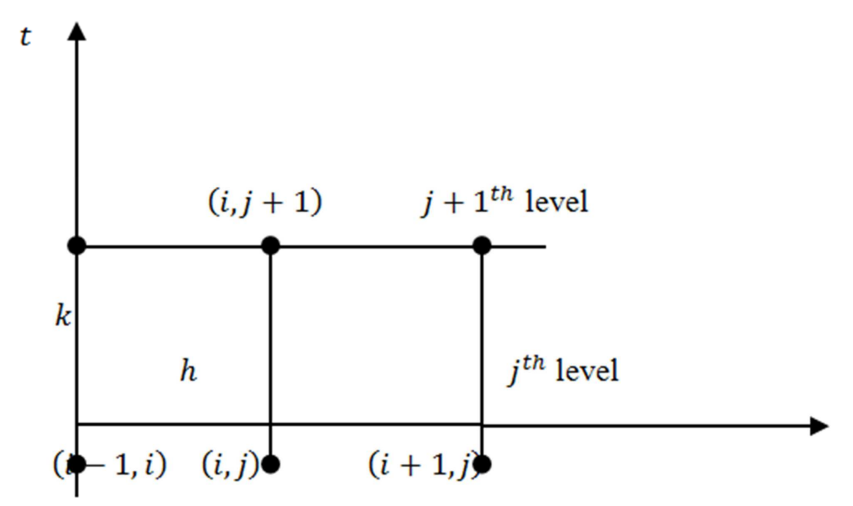

Figure 2. Schematic representation.

Recall that the heat equation in one dimension, i.e., $u_{t}-C^{2} U_{x x}=0$ where $C^{2}$ is diffusibility of substance we have approximation for the derivative.

$$
\frac{\partial u}{\partial t}=\frac{u_{i}^{j+1}-u_{i}^{j}}{k} \text { and } \frac{\partial^{2} u}{\partial x^{2}}=\frac{u_{i-1}^{j}-2 u_{i}^{j}+u_{i+1}^{j}}{h^{2}}
$$

By substituting these, we obtain

$$
u_{i}^{j+1}-u_{i}^{j}=\frac{k c^{2}}{h^{2}}\left[u_{i-1}^{j}-2 u_{i}^{j}+u_{i+1}^{j}\right]
$$

If $\alpha=k c^{2} / h^{2}$ is mesh ratio parameter

$$
u_{i}^{j+1}=\alpha u_{i-1}^{j}+(1-2 \alpha) u_{i}^{j}+\alpha u_{i+1}^{j}
$$

which is two level explict formula.

Note: The above formula enables us to determine the value of $\mathrm{u}$ at $(i, j+1)^{\text {th }}$ mesh point in terms of the known function values at the point $x_{i-1}, x_{i}$ and $x_{i+1}$ at the instant $t_{i}$.

In particular, when $\alpha=\frac{1}{2}$ the above formula becomes

$$
u_{i}^{j+1}=\frac{1}{2}\left(u_{i-1}^{j}+u_{i+1}^{j}\right) \text {. }
$$

Which shows that values of $u$ at $x_{i}$ at time $t_{i+1}$ is the mean of the $u$ values at $x_{i-1}$ and $x_{i+1}$ at time $t_{j}$.

As you can observe from (10) Schmidt method is valid only for $0<\alpha \leq \frac{1}{2}$, see, e.g []

Now, Let us solve

$$
\begin{gathered}
\frac{\partial u}{\partial t}-4 \frac{\partial^{2} u}{\partial x^{2}}=0 \\
u(0, t)=0=u(8, t) \text { and } u(x, 0)=4 x-\frac{x^{2}}{2} \\
\text { At the points } x_{i}=i: i=0,1,2, \ldots, 8 \text { and } t_{j}=\frac{1}{8} j: j=
\end{gathered}
$$
$0,1,2, \ldots, 5$

Solution:

Given that $C^{2}=4, h=1$ and $k=\frac{1}{8}$

Then $\alpha=C^{2} k / h^{2}=1 / 2$

Thus we have

$$
u_{i}^{j+1}=\frac{1}{2}\left(u_{i-1}^{j}+u_{i+1}^{j}\right)
$$

Because $u(0, t)=0=u(8, t), U_{0}^{j}=0$ and $U_{8}^{j}=0 \forall j$, i.e. the entries in the first and last column of table 1 are zero. Since

$$
\begin{gathered}
u(x, 0)=4 x-\frac{x^{2}}{2} \\
u_{1}^{0}=4 i-\frac{1}{2} i^{2}=0,3,5,6,7,5,8,7,5,6,3,5 \text { for } i \\
=0,1,2, \ldots 7 \text { at } t=0
\end{gathered}
$$

respectively. These are the entries in the first row of table 1 putting $j=0$, in the above formula, we have

$u_{1}^{1}=\frac{1}{2}\left(u_{i-1}^{0}+u_{i+1}^{0}\right)$ and taking $i=1,2, \ldots 7$ successively

We get $u_{1}^{1}=3, u_{2}^{1}=5.5, u_{3}^{1}=7, u_{4}^{1}=7.5, u_{5}^{1}=7, u=$ 5.5 and $u_{7}^{1}=3$

Putting $j=1$ in above formula, the entries of the third row are given by:

$u_{1}^{2}=\frac{1}{2}\left(u_{i-1}^{1}+u_{i+1}^{1}\right)$ and taking $i=1,2, \ldots 7$ successively

We get $u_{1}^{2}=2.75, u_{2}^{2}=5, u_{3}^{2}=6.5, u_{4}^{2}=7, u_{5}^{2}=$ $6.5, u_{6}^{2}=5$ and $u_{7}^{2}=2.75$

Similarly putting $j=2,3,4$ successively in above formula we have the entries of the fourth and sixth rows of table. Hence the value of $u_{i}^{j}$ are given as follow:

Table 1. The value of $u$ at $x_{i}$ and $t_{j}$ for $i=0,1,2, \ldots, 8$ and $j=0,1,2, \ldots, 5$.

\begin{tabular}{llllllllll}
\hline $\boldsymbol{i}$ & $\boldsymbol{j}$ & $\mathbf{0}$ & $\mathbf{1}$ & $\mathbf{2}$ & $\mathbf{3}$ & $\mathbf{4}$ & $\mathbf{5}$ & $\mathbf{6}$ & $\mathbf{7}$ \\
\hline 0 & 0 & 3.5 & 6 & 7.5 & 8 & 7.5 & 6 & 3.5 & 0 \\
1 & 0 & 3 & 5.5 & 7 & 7.5 & 7 & 5.5 & 3 & 0 \\
2 & 0 & 2.75 & 5 & 6.5 & 7 & 6.5 & 5 & 2.75 & 0 \\
3 & 0 & 2.5 & 4.625 & 6 & 6.5 & 6 & 4.625 & 2.5 & 0 \\
4 & 0 & 2.3125 & 4.25 & 5.5625 & 6 & 5.5625 & 4.25 & 2.3125 & 0 \\
5 & 0 & 2.125 & 3.9375 & 5.125 & 5.5625 & 5.125 & 3.9375 & 2.125 & 0 \\
\hline
\end{tabular}


Hence, the computed solutions for few time steps are shown above. The solution at any point $(i, j+1)$ is the average of the two adjacent mesh points $(i-1, j)$ and $(i+$ $1, j)$ of the previous time step. As we observed from the table we note that as $t$ increases down the column, the solution decreases. This is accordance known exact solutions of heat conduction problem which we observe practically in life.

Further the computed solution lies between 0 and 8 .

From fore going discussion, Let us see what happen to the solution if we reduce $t$ level from $\frac{1}{8}$ to $1 / 64$ and $x$ level from 1 to $1 / 2$

Let us find the solution at the point $x_{i}=\frac{i}{2}: i=0,1,2, \ldots 16$ and $t_{j}=\frac{1}{64} j: j=0,1,2,3,4$, of above problem.

Solution: Given $C^{2}=4, h=\frac{1}{2}$ and $k=\frac{1}{64}$

Then $\alpha=\frac{C^{2} k}{h^{2}}=\frac{1}{4}$

Because $u(0, t)=0=u(8, t), U_{16}^{1}=0$ for all values of $j$, i.e., the entries in the first and last column of table 2 are zero.

$$
\begin{aligned}
& \text { Since } u(x, 0)=4 x-\frac{x^{2}}{2} \\
& u_{1}^{0}=\frac{4 i}{2}-\frac{1}{2}\left(\frac{i}{2}\right)^{2} \text { for } i=0,1,2, \ldots, 16 \text { and } t=0 \\
& =2 i-0.125 i^{2}, i: i=0,1,2, \ldots, 16 \text { and } j=0
\end{aligned}
$$

These are the entries of the first row in the table 2 .

Now, from forward time central space formula, i.e. The
Schmidt method; we have

$$
\begin{aligned}
u_{1}^{j+1}=\frac{1}{4}\left[u_{i-1}^{j}+\right. & \left.2 u_{i}^{j}+u_{i+1}^{1}\right], i: i=1,2,3, \ldots, 15 \text { and } j: j \\
= & 1,2,3, \ldots
\end{aligned}
$$

Putting $i=0$, we have

$$
u_{i}^{1}=\frac{1}{4}\left[u_{i-1}^{0}+2 u_{1}^{0}+u_{i+1}^{0}\right]
$$

Thus, by taking $i=1,2,3, \ldots, 15$ successively, we get those entries of the third row in the table 2 .

Putting $j=2$, we have

$$
u_{i}^{3}=\frac{1}{4}\left[u_{i-1}^{2}+2 u_{1}^{2}+u_{i+1}^{1}\right]
$$

Thus, by taking $i=1,2,3, \ldots, 15$ succesively, we get those entries of the fourth row in the table 2 .

\begin{tabular}{|c|c|c|c|c|c|c|c|c|c|}
\hline $\boldsymbol{i} \boldsymbol{j}$ & $\mathbf{0}$ & 1 & 2 & 3 & 4 & 5 & 6 & 7 & 8 \\
\hline 0 & 0 & 1.875 & 3.5 & 4.875 & 6 & 6.875 & 7.5 & 7.875 & 8 \\
\hline 1 & 0 & 1.8125 & 3.4375 & 3.4375 & 4.8125 & 5.9375 & 6.8125 & 7.4375 & 7.8125 \\
\hline 2 & 0 & 1.7656 & 3.375 & 4.75 & 5.875 & 6.75 & 7.375 & 7.75 & 7.875 \\
\hline 3 & 0 & 1.7266 & 3.3164 & 4.6875 & 5.8125 & 6.6875 & 7.3125 & 7.6875 & 7.8125 \\
\hline 4 & 0 & 1.3174 & 3.2617 & 4.626 & 5.75 & 6.625 & 7.25 & 7.625 & 7.75 \\
\hline
\end{tabular}

Putting $j=3$, we have

$$
u_{i}^{4}=\frac{1}{4}\left[u_{i-1}^{3}+2 u_{1}^{3}+u_{i+1}^{3}\right]
$$

Thus, by taking $i=1,2,3, \ldots, 15$ successively, we get those entries of the last row in the table 2 . Therefore, let us see the solution in the table 2 .

Table 2. The value of $u$ at $x_{i}$ and $t_{j}$ for $i=0,1,2, \ldots 16$ and $j=0,1,2,3,4$.

Table 2. Continued.

\begin{tabular}{llllllll}
\hline $\boldsymbol{i} \boldsymbol{j}$ & $\mathbf{9}$ & $\mathbf{1 0}$ & $\mathbf{1 1}$ & $\mathbf{1 2}$ & $\mathbf{1 3}$ & $\mathbf{1 4}$ & $\mathbf{1 5}$ \\
\hline 0 & 7.875 & 7.5 & 6.875 & 6 & 4.875 & 3.5 & \\
1 & 7.9375 & 7.8125 & 7.4375 & 6.8125 & 5.9375 & 4.8125 \\
2 & 7.75 & 7.375 & 6.75 & 5.875 & 4.75 & 1.875 & 3.375 \\
3 & 7.6875 & 7.3125 & 6.6875 & 5.8125 & 4.6875 & 3.8125 & 0 \\
4 & 7.625 & 7.25 & 6.625 & 5.75 & 4.626 & 0 & 3.2616 \\
\hline
\end{tabular}

\subsection{Comparison Between Analytical and Numerical Methods for One Dimension Heat Equation}

In the previous section, we obtained the analytical and numerical solution of the given problem of heat flow in one dimension.

\begin{tabular}{|c|c|c|c|c|c|c|c|c|c|}
\hline$x_{1}$ & $\mathbf{0}$ & 1 & 2 & 3 & 4 & 5 & 6 & 7 & 8 \\
\hline 0 & 0 & 3.4979 & 5.9987 & 7.4989 & 7.9990 & 7.4989 & 5.9987 & 3.4979 & 0 \\
\hline 0.125 & 0 & 3.0754 & 5.508 & 7.002 & 7.5000 & 7.002 & 5.5058 & 3.0754 & 0 \\
\hline 0.25 & 0 & 2.7799 & 5.0568 & 6.5100 & 7.0016 & 6.5100 & 5.0568 & 2.7799 & 0 \\
\hline 0.375 & 0 & 2.5426 & 4.6594 & 6.03808 & 6.5110 & 6.03808 & 4.6594 & 2.5426 & 0 \\
\hline 0.5 & 0 & 2.3387 & 4.3003 & 5.5963 & 6.0788 & 5.5963 & 4.3023 & 2.3387 & 0 \\
\hline 0.625 & 0 & 2.1577 & 3.9773 & 5.1842 & 5.6057 & 5.1842 & 3.9773 & 2.1577 & 0 \\
\hline
\end{tabular}

Recall that the analytical solution which we obtained by adding 8 terms of the infinite series is given in the table 3 whereas, the numerical solution which we obtained at the same value of $x$ and $t$ of analytical solution is given in the table 4 .

So, Let us see the solution in the table 3 and table 4 as the following.

Table 3. The analytical solution. 
Table 4. The numerical solution obtained at $x_{i}=i: i=0,1,2, \ldots, 8$ and $t_{j}={ }^{j} / 8: j=0,1,2,3,4,5$.

\begin{tabular}{lllllllllll}
\hline $\boldsymbol{i}$ & $\boldsymbol{j}$ & $\mathbf{0}$ & $\mathbf{1}$ & $\mathbf{2}$ & $\mathbf{3}$ & $\mathbf{4}$ & $\mathbf{5}$ & $\mathbf{6}$ & $\mathbf{7}$ & $\mathbf{8}$ \\
\hline 0 & 0 & 3.5 & 6 & 7.5 & 8 & 7.5 & 6 & 3.5 & 0 \\
1 & 0 & 3 & 5.5 & 7 & 7.5 & 7 & 5.5 & 3 & 0 \\
2 & 0 & 2.75 & 5 & 6.5 & 7 & 6.5 & 5 & 2.75 & 0 \\
3 & 0 & 2.5 & 4.625 & 6 & 6.5 & 6 & 4.625 & 2.5 & 0 \\
4 & 0 & 2.3125 & 4.25 & 5.5625 & 6 & 5.5626 & 4.25 & 2.3125 & 0 \\
5 & 0 & 2.125 & 3.9375 & 5.125 & 5.5625 & 5.125 & 3.9375 & 2.125 & 0 \\
\hline
\end{tabular}

As you can observe from both above table, if the time is large and large, the magnitude of heat distributed in the material is tends to generate equal heat distribution.

Now, let us take any row from both tables. For instance,

$$
\begin{aligned}
& u\left(x_{1}, 0.125\right)= \\
& \text { (03.0754 5.508 7.002 7.500 7.002 5.5058 3.0754 0) (a) } \\
& \text { and } u_{i}^{1}=\left(\begin{array}{lll}
0 & 35.577 .575 .530
\end{array}\right)(b) \\
& \text { for } i: i=0,1,2, \ldots, 8
\end{aligned}
$$

Note that $U_{i}^{1}$ are the values of $u$ at $t_{1}=\frac{1}{8}=0.125$

From row (a): the total magnitude of heat at $t_{1}=$ 0.125 is 38.6686 and from row (b): the total magnitude of heat at $t_{1}=0.125$ is 38.5 . This indicate that the total magnitude of heat which approximated by analytical and numerical approach is nearly the same.

Furthermore, from above two rows (fixing time value) as you see, each respective value is the same up to first decimal places. In both row as we see from center to the boundary point the solution decreases. At boundary point both numerical and analytical solution is zero which satisfies the boundary condition we set previously. Furthermore, fixing the value of $x$ at 1 (varying the time value), we produce the following graphs which compares the analytical and Numerical solution.

Comparison between Analytical and Numerical solution at $x=1$.

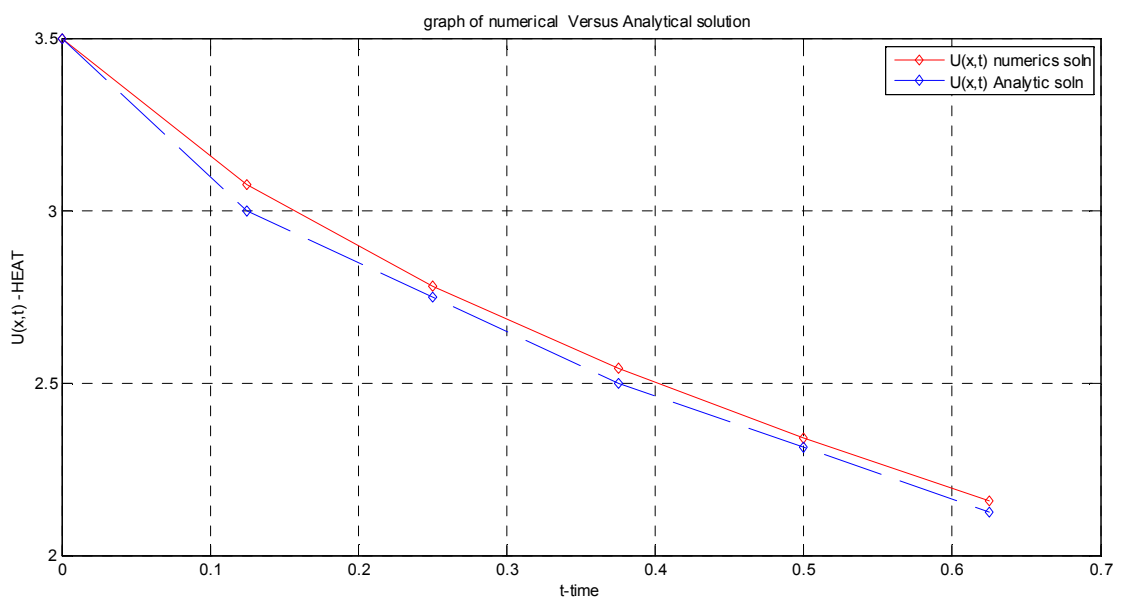

Figure 3. Comparison between analytical and Numerical Solution.

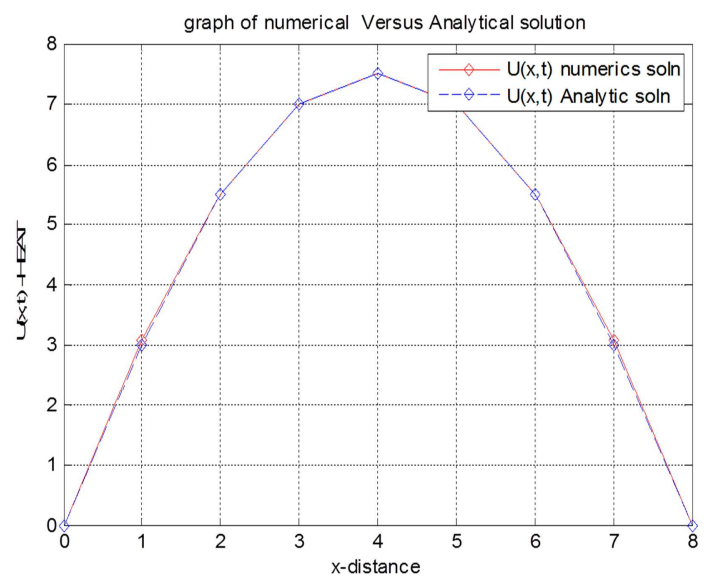

Figure 4. Comparison between Analytical and Numerical solution at $t=0.125$.
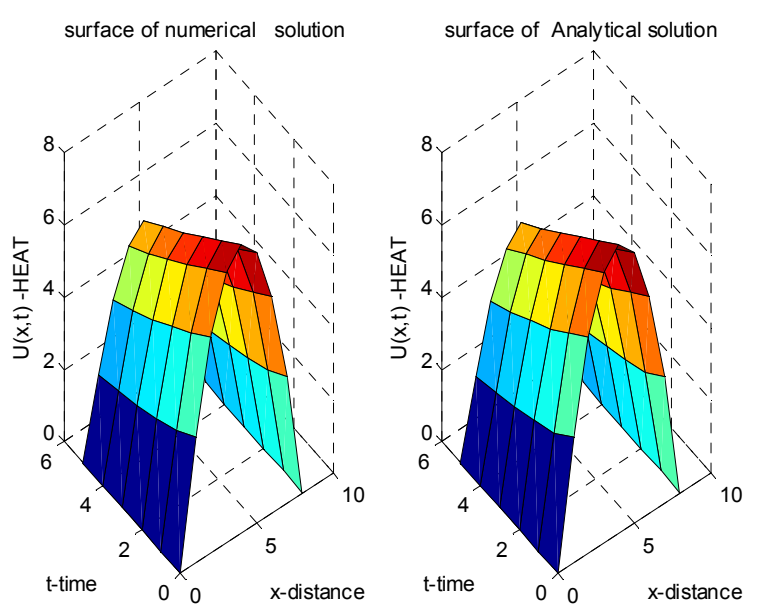

Figure 5. Comparison of surface between Analytical and Numerical solution of heat equation. 


\section{Discussion}

We understand from above solution that heat will flow from higher potential to lower potential until equilibrium is generated. At $x=0$ and $x=8$ the above solution is zero. i.e., the solution satisfies the given boundary condition.

Now, let us recall, from analytical solution

$$
u_{n}(x, t)=\frac{256}{\pi^{3}} \frac{1}{(2 n-1)^{3}} \sin \frac{(2 n-1) \pi x}{8} e^{\frac{-(2 n-1)^{2}}{16} \pi^{2} t}
$$

This implies

$$
\left|u_{n}(x, t)\right|=\left|\frac{256}{\pi^{3}(2 n-1)^{3}} \sin \frac{(2 n-1) \pi x}{8} e^{\frac{-(2 n-1)^{2}}{16} n^{2} t}\right| \leq \frac{256}{\pi^{3}(2 n-1)^{3}}
$$

Thus, for any $\mathrm{n}$ terms could be taken to get the analytical solutions of the above problem,

$$
u(x, t) \leq \frac{256}{\pi^{3}} 0 \leq x \leq 8 \text { and } t \geq 0 .
$$

Also, the analytical solution we obtained satisfies this criteria. Even so, if you add any $n$ terms which is greater than six and if you correct the solutions up to fourth decimal places, The result is the same as above solution in table 3 for the same values of $x$ and $t$.

As we observed from the numerical solution, if $\Delta t$ and $\Delta x$ is reduced, the numerical method is best approximates the solution. That is the more nodes taken, the closer approximate the solution. Thus, the finite difference solution is sensitive to the value of $\alpha$ which involves the mesh lengths In addition, we understand that as the time step increases the distribution of heat decreases within the material.

We obtained the analytical solution which involves such infinite series solution that its evaluation becomes exceedingly difficult. It is worthwhile to mention here that analytical solutions are not always possible to obtain; indeed, in many instances they are very cumbersome and difficult to use. In this case numerical methods are used more advantageous than analytical methods. However, the analytical approach has its own advantage. If you add a lot of terms of the series the analytical approach is better approximate the solution.

\section{Conclusion}

This paper tries to set the procedure on how to solve heat equation in one dimension using Analytical and Numerical methods and the solution found to be in a good agreement. That is, the solution agrees up to one decimal place. Note that by describing the domain (dividing into increments in the both $x$ and $t$ direction where $i$ location indicate the $x$ increment and $j$ location indicate the time increment) we get the temperature value at any nodal points. Furthermore, though the analytical approach is good in finding the exact solution to the problem, it is difficult to manipulate as it involves an infinite series. The Numerical approach we used though simple it is an approximation and we need to go more iteration to be in agreement with reality. Furthermore, advantage of an explicit forward finite difference we used is the direct calculation of future nodal temperature. However, stability of this solution governed by selection values of $\Delta x$ and $\Delta t$.

\section{References}

[1] Yehude Pinch over and Jacob Rubinstein, an introduction to partial differential equation, 2005.

[2] Steven Chapra Raymond P Canale, numerical methods for engineers, $7^{\text {th }}$ edition, 2015.

[3] J. P. Holman, "Heat transfer", tenth edition, 2010.

[4] David Borthwick, 'introduction to partial differential equation and it's application', $1^{\text {st }}$ edition, 2016.

[5] Aslak Tveito, Ragnar Winther, 'introduction to partial differential equation and it's application: a computational approach' 2015.

[6] Mehta, Nirajkumar \& Vipul, Mr \& Gondaliya, Bhavesh \& Jayesh, Mr \& Gundaniya, V. (2013). Applications of Different Numerical Methods in Heat Transfer - A Review. International Journal of Emerging Technology and Advanced Engineering. 3. 363 .

[7] Edmund Agyeman, Derick Folson, International Journal of Computer Applications (0975 - 8887) Volume 79 - No5, October 2013

[8] Matthew J. Hancock, The 1-D Heat Equation, 2006

[9] Vitoriano Ruas, Numerical Methods for Partial Differential Equations: An Introduction, 2016

[10] Ahmad, Najmuddin \& Charan, Shiv. (2018). STUDY OF NUMERICAL ACCURACY OF ONE DIMENSIONAL HEAT EQUATION BY BENDER-SCHMIDT METHOD, CRANK-NICHOLSON DIFFERENCE METHOD AND DU FORT AND FRANKEL METHOD.

[11] Walter A. Strauss, partial differential equations, an introductions, 2008.

[12] Subani, Norazlina, Faizzuddin Jamaluddin, Muhammad Arif Hannan Mohamed, and Ahmad Danial Hidayatullah Badrolhisam. "Analytical Solution of Homogeneous OneDimensional Heat Equation with Neumann Boundary Conditions." In Journal of Physics: Conference Series, vol. 1551, no. 1, p. 012002. IOP Publishing, 2020.

[13] Lapidus, Leon, and George F. Pinder. Numerical solution of partial differential equations in science and engineering. John Wiley \& Sons, 2011. 
[14] Agyeman, Edmund, and Derick Folson. "Algorithm analysis of numerical solutions to the heat equation." International Journal of Computer Applications 79, no. 5 (2013).

[15] NIGATIE, Y. "The finite difference methods for parabolic partial differential equations." Journal of Applied \& Computational Mathematics 7, no. 3 (2018): 1-4.

[16] Mohamad, A. N., and Sisay Fikadu. "EFFECT AND EFFICIENT APPROACH OF SOLVING HEAT EQUATION
BY DIFFERENT NUMERICAL APPROACHES INCLUDING CRANK-NICOLSON SCHMIDT (METHOD) WHICH IS STILL ACTING AS A MAJOR ROLE." International Journal of Advanced Research in Engineering and Applied Sciences 6, no. 7 (2017): 18-41.

[17] Makhtoumi, Mehran. "Numerical solutions of heat diffusion equation over one dimensional rod region." arXiv preprint arXiv: 1807.09588 (2018). 